\title{
Optical Diffuse Light in Nearby Compact Groups
}

\author{
Cláudia Mendes de Oliveira ${ }^{1}$, Cristiano Da Rocha ${ }^{1}$, Carlos R. Rabaça ${ }^{2}$, Daniel \\ N. E. Pereira ${ }^{2}$, and Michael Bolte ${ }^{3}$ \\ 1 Instituto de Astronomia, Geofísica e Ciências Atmosféricas, U. de São Paulo, Brazil \\ 2 Obs. do Valongo, U. Federal do Rio de Janeiro, Brazil \\ 3 Lick Observatory, Board of Studies in Astronomy and Astrophysics, U. of \\ California, Santa Cruz, USA
}

\begin{abstract}
Analyses of B and R band observations of four compact groups reveal the presence of a considerable amount of diffuse, intergalactic light in two of them (HCG 79 and HCG 95). The diffuse component is presumably due to stellar material that has been tidally stripped from the galaxy group members. A new approach is used to measure this diffuse background light, using wavelet techniques for detecting low surface brightness signals. The diffuse light component has a mean colour of $(B-R)$ $=1.4-1.5 \pm 0.1$ and it comprises the following fractions of the total group light in the B band: $18 \%, 12 \%, 3 \%$ and 0\% for groups HCG 95, HCG 79, HCG 92 and HCG 88, respectively. The diffuse light content of a group may represent an efficient tool for the determination of how long groups have been together in a compact configuration.
\end{abstract}

\section{Introduction}

A simple visual inspection of compact groups of galaxies from Hickson's catalogue (Hickson 1982) shows that several of them contain diffuse background light. This diffuse component is presumably due to stellar material that has been tidally stripped from the galaxy group members. The structure of the background light is, however, often obscured by the light from the group galaxies. We have developed a new method to isolate the contribution from the background light in groups by using the à trous wavelet method. We apply it for four compact group galaxies and describe the preliminary results in the present contribution.

\section{Sample and Analysis}

We studied a subsample of four groups from the Hickson compact group catalogue (Hickson 1982). The data for this analysis come from $B$ and $R$ images obtained at different telescopes and instruments. Table 1 summarizes the observations.

Details of each group studied are given in the following.

- HCG 79 or Seyfert's sextet: This is one of the most famous, as well as the densest, compact group of galaxies. It is composed of four galaxies at a redshift of $\mathrm{z}=0.0145$ and one foreground galaxy at $\mathrm{z}=0.0661$. 
- HCG 88: This group is composed of four spiral galaxies lying along a line as projected on the sky, with a $2 \mathrm{D}$ velocity dispersion of only $26 \mathrm{~km} \mathrm{~s}^{-1}$. It is at $\mathrm{z}=0.0201$.

- HCG 92 or Stephan's quintet: the core of this group is composed of one elliptical system and three spiral galaxies at a mean redshift of $\mathrm{z}=0.0215$. The fifth galaxy of the quintet is a foreground galaxy with a velocity of $\sim$ $800 \mathrm{~km} / \mathrm{s}$. One of the four accordant members of the group (NGC 7318B) has a velocity that is $\sim 1000 \mathrm{~km} / \mathrm{s}$ higher than the mean for the other three galaxies of the group. Strong tidal filaments are observed in this group.

- HCG 95: this quartet is the most distant group in our sample, at a redshift of $\mathrm{z}=0.0396$. One of the members of this group is an on-going gas-rich merger with two tidal tails, which may be, in addition, in interaction with the elliptical galaxy of the group (NGC 7609).

The images were reduced using IRAF and they were then analyzed using a signal processing technique based on the à trous wavelet transform. Such a technique (multiscale vision model, Bijaoui and Rué 1995) is well suited for analyzing complex systems composed of structures with different sizes. An example of various "levels" of the wavelet analysis is shown in Fig. 1

Fig. 1. B image of HCG 79 decomposed into six "levels" with the à trous algorithm. Structures with different size are seen in each level. The panels show the detected and reconstructed objects at each characteristic scale, from small to large, ordered from left to right and from top to bottom.

Table 1. Observations of compact groups

\begin{tabular}{|c|c|c|c|c|c|}
\hline Group & Tel/Instr. & $\begin{array}{l}\operatorname{Exp}(B, R) \\
\text { seconds }\end{array}$ & $\begin{array}{l}\% \text { of B } \\
\& \text { R light }\end{array}$ & $\begin{array}{l}<\mathrm{SB}_{B}> \\
\operatorname{mag}\end{array}$ & B-R \\
\hline HCG 79 & CFHT/SIS & 2700,1800 & $13 \pm 1,12 \pm 1$ & $25.5 \pm 0.1$ & $1.5 \pm 0.1$ \\
\hline HCG 88 & CFHT/MOS & 3600,4800 & $0, \quad 0$ & - & - \\
\hline HCG 92 & Gemini/GMOS & $900, \quad-$ & $3 \pm 2, \quad-$ & - & - \\
\hline HCG 95 & CFHT/MOS & 2400,3000 & $18 \pm 1,18 \pm 1$ & $26.2 \pm 0.1$ & $1.4 \pm 0.1$ \\
\hline
\end{tabular}

\section{Results}

Our preliminary results are summarized in columns 4 to 6 of Table 1 . The percentage of the total group light that is in the background halo of diffuse light is 
listed in column 4, for the B and $\mathrm{R}$ band images. The mean surface brightness and colour of the background light are listed in columns 5 and 6 .

The errors were obtained based on simulations of galaxies and background light (with different shapes) using the task artdata in IRAF. Following our simulations, we were able to determine errors for the surface brightnesses, magnitudes, and fraction of total light in the diffuse background component.

\section{Notes on individual groups}

Fig. 2 shows a B image of the group HCG 79 with the contour plots of the diffuse light overplotted (the shape of the diffuse light component for the $\mathrm{R}$ image is very similar to that measured in the B image). The diffuse light profile of this group is irregular at all radii. The colour of the diffuse light is similar to the colours of the outskirts of the galaxies (or colours of low-luminosity galaxies which could possibly have been stripped). It is interesting to note that the HI contours (Williams et al. 1991) of HCG 79 show similar shape to the optical diffuse light envelope.

Fig. 2. B image of H79 with superimposed diffuse light intensity curves (continuous lines). The brightest contours are, from the center outwards, 25.0, 25.25, 25.5, 25.9, 26.4 and $27.5 \mathrm{mag} / \operatorname{arcsec}^{2}$. The center of the diffuse light component and the geometric center of the group are shown with " $\times$ " and "+", respectively.

For HCG 95, the optical diffuse light we detected represented $18 \%$ of the total light of the group and for HCG 88, we could detect no optical diffuse light.

The analysis of an $\mathrm{R}$ image of the Stephan quintet, obtained with Gemini in July 2002 shows that although there are several tails and other sharp lowluminosity features, there does not seem to exist an envelope of light around the

group. Instead, the diffuse background light is concentrated in a position north of the pair NGC $7318 \mathrm{~B} / \mathrm{D}$, in between the two northern tails which emanate from these galaxies.

\section{Summary, Conclusion and Perspectives}

Previous claims for a lack of diffuse optical light in compact groups (Rose 1979; Pildis, Bregman, \& Schombert 1995) were based on data insensitive to low surface brightness features or not fully processed to reveal them. Our analysis of the $B$ and $R$ images of four compact groups using wavelet analysis indicated the presence of background light in three of them.

Important information about the influence of interaction processes in galaxy evolution can be provided by the detection of diffuse light. Compact groups have a high surface density compared to the field and most of the $N>4$ groups are 
probably bound and virialized. The presence of light that cannot be explained by the overlapping envelopes of individual galaxies indicates that tidal encounters have already stripped the galaxies of significant mass. The stripped material should be distributed in the group potential in the form of a diffuse intergalactic medium of stars and clusters. The presence of significant halos around dense systems constitute proof that the galaxies have been interacting, that they have had significant dynamical evolution and provides a test of group models.

In the particular case of the two groups with the largest amount of diffuse light studied here, the presence of a diffuse halo indicates that the member galaxies have been together for at least a few crossing times. On the other hand, the irregular shapes of the diffuse light envelopes suggest that the members have been together for a relatively short timescale.

We plan to combine the measures of the fraction of the total light in diffuse halos and the shapes of the halos with n-body predictions in order to obtain estimates for the dynamical ages of dense groups.

\section{Acknowledgments}

C.M.dO. and C.D.R. would like to acknowledge funding from FAPESP (grant No. 96/08986-5). They also thank PRONEX, the Alexander von Humboldt Foundation, ESO and the conference organizers for making possible the attendance to the conference. CRR acknowledges funding from FAPERJ (E-26/171.085-99). We are grateful to Laerte Sodré Jr. and Eduardo Cypriano for the joint Gemini proposal of HCG 92 .

\section{References}

1. A. Bijaoui, F. Rué: Signal Processing 46, 345 (1995) W. Frank, A. Seeger: Appl. Phys. A 3, 66 (1988)

2. P. Hickson: ApJ 255, 382 (1982)

3. R. A. Pildis, J.N. Bregman, A.E. Evrard: ApJ 443, 514 (1995)

4. R.A. Pildis, J.N. Bregman, J.M. Schombert: AJ 110, 1498 (1995)

5. J.A. Rose: ApJ 231, 10 (1979)

6. B.A. Williams, P.M. McMahon, J.H. van Gorkom: AJ 101, 1957 (1991) 
This figure "f1.jpg" is available in "jpg" format from: http://arxiv.org/ps/astro-ph/0210576v2 
This figure "f2.jpg" is available in "jpg" format from: http://arxiv.org/ps/astro-ph/0210576v2 